\title{
Disturbance Force Estimation for a Low Pressure Suction Gripper Based on Differential Pressure Analysis.
}

\author{
Benjamin Wirth ${ }^{1}$, Sven Coutandin ${ }^{1}$ and Jürgen Fleischer ${ }^{1}$ \\ ${ }^{1}$ Karlsruhe Institute of Technology, 76131 Karlsruhe, Germany \\ benjamin.wirth@kit.edu \\ sven. coutandin@kit.edu \\ juergen.fleischer@kit.edu
}

\begin{abstract}
In times of ever increasing performance requirements imposed on newly developed products, fiber reinforced plastics have become essential for lightweight applications. While mass related stiffness and strength are huge advantages, automatic handling of the raw materials, such as fabrics, can be very challenging. Low-pressure suction grippers have been proven to be capable of handling fragile air-permeable materials in a non-destructive manner, giving them a clear advantage over potentially damaging competing gripping technologies such as needle grippers. However, they also consume large amounts of energy to ensure successful handling operations. This research aims to estimate disturbance forces applied to the manipulated workpiece by monitoring its internal suction pressure, which will in turn allow for running the grippers at a bare minimum power while still being able to react to disturbances enabling safe handling operations.
\end{abstract}

Keywords: Process Monitoring, Gripping Technology, Textile Handling.

\section{Introduction}

While weight related stiffness and strength of continuous-fiber reinforced plastics (FRP) are superior to most classic metallic materials [1]. There is a lack of automation solutions in the handling of "limp and air-permeable semi-finished textile products" [2]. Low pressure suction grippers have been identified as a technology with great potential for automated handling of semi-finished textile materials [3, 4].

They can maintain a moderate suction pressure at high volumetric flow rates [5], which allows for sufficient holding forces even when air is able to pass into the suction cavity of the gripper either by leaking airstreams passing the material or by penetrating the porous material directly.

For automated handling of air-permeable sheet materials, low pressure suction grippers have been used in a variety of research applications [6-9].

However, due to a lack of compensation mechanisms for 'disturbances during the handling process' they are often run at maximum suction power to ensure reliable material transportation even when exterior forces may interact with the handled textiles. This mode of operation is very energy-inefficient and thus not desirable. [10] 
This work focuses on estimation of disturbing forces applied during handling of airpermeable blanks by monitoring the interior pressure inside the grippers' central cavity.

\section{Low Pressure Suction Grippers}

Grippers using partial vacuums at moderate volumetric flow rates have been identified as one of the most promising technologies for automated textile handling [11].

Fig. 1 shows a sketch for a simple suction gripper.

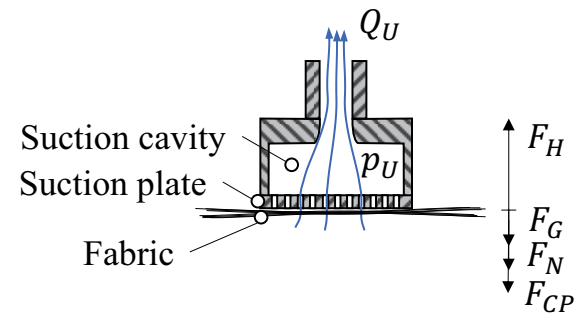

Fig. 1. Sketch of a simple suction gripper and Forces acting on a gripped Fabric. Based on [10]

These systems usually feature central cavities, which are partially evacuated to a low pressure throughout this paper the differential pressure between suction cavity and surrounding atmosphere will be depicted by $p_{U}$.

A suction plate, which may contain small holes preventing major material deformations and restricting airflow in non-covered areas, is usually attached to the gripper. This avoids "short circuits", that would lead to a rapid decline in suction pressure $p_{U}$ [12].

In a one dimensional lifting operation the handled materials are generally subjected to four different forces [10]:

- $F_{H}$, a holding Force exerted by the pressure drop across a gripped sheet. An equation formulated by Götz relates the holding force exerted on the gripped material $F_{H}$ to the pressure-drop $\Delta p$ across the material by definition of an effective area $A_{\text {eff }}$. [12]

$$
F_{H}=A_{e f f} \cdot \Delta p
$$

- $F_{G}$, the force excerted on the object by gravity.

- $F_{N}$, a combination of other dynamically acting forces in normal direction. Such as inertial forces or external forces these will hereinafter be called disturbances or disturbance forces.

- $F_{C P}$, a constraining force applied by the suction plate contacting the fabric.

The contact force with which any gripped material is pressed against the suction plate can be described by [10]:

$$
F_{C P}=F_{H}+F_{G}+F_{N}
$$




\section{Evaluation of the State of the Art}

\subsection{Pressure Monitoring for Suction Grippers}

Götz measured differential pressures inside a low pressure handling system for leather blanks during operation for validation purposes, however, he did not relate them to significant process properties. [9]

The gauge pressure inside a suction Gripper has been used for gripper control by Ehinger. The interior pressure was controlled by a PI-controller to match predefined values found by experimental analysis. Allowing for secure draping of a textile material even with some non-covered holes on the suction plate. [6] While controlling the interior pressure will lead to a response on disturbing forces acting on the handled material, these forces are not quantified. For that an additional observation of the supplied suction energy would be necessary.

Fleischer et al. have shown a dependence of the internal negative pressure inside a coanda-effect based grippers' suction cavity on the number of manipulated layers for air-permeable materials. In experiments placing up to 2 layers of textile materials beneath a suction plate and gradually decreasing the input power-stream to the gripper it was found, that for any given input power the interior pressure approaches a higher vacuum with additional layers adhering to the manipulator. This allows the system to directly estimate the number of sheets separated from a stack-magazine. [8]

\subsection{Gripping Force Estimation for Low Pressure Suction Grippers}

Fleischer et al were able to measure holding forces between a suction gripper and carbon fiber materials by attaching brass electrodes to the suction plate. The measured electrical resistance between the electrodes has been shown to directly depend on brasscarbon contact resistances, which in turn decrease with higher holding forces applied to the handled textile. By designing control loops for energy efficient operation, they were able to create a dynamic system capable of quickly reacting to disturbances, while also reducing energy consumption by nearly $90 \%$ compared to a conventional static energy supply. Furthermore, they were able to control the holding force, such that a reliable separation of singular sheets off of a stack-magazine was possible. [8]

This approach, however, relies on the electrical conductivity of the handled textile and is therefore not applicable for a wide variety of common materials.

Brink et al. developed a "Force Controlled Variable Fan Gripping Module" incorporating an electrically powered fan as a vacuum generator. Strain gauges were integrated into the fan grill, which serves as a suction plate in this system. With increasing handling forces, increasing deformations in the grill can be observed, which were in turn registered as a change in resistance for the strain gauges. Using this principle, Brink et al. were able to monitor process forces occurring during the handling of non-crimp fabrics. They furthermore noted, that a dependence of the gripping force on the number of attached layers is observable. [7] 
Retrofitting sensor integrated suction plates introduces undesired complex alterations to commercial gripping-systems. A less invasive approach for measuring disturbances acting on the gripper is required. Monitoring the pressure inside the suction cavity promises to be an efficient solution to this problem. This research shows an approach for generation of a process model relating negative pressure to disturbance forces based on an offline calibration. This system can be used for online monitoring of handling operations. It may also be used as part of a state estimation algorithm allowing the gripper system to control contact forces at the gripper-material interface.

\section{System Description}

The SCG 1xE100 gripper used in this research (see Fig. 2) is manufactured by J. Schmalz GmbH.
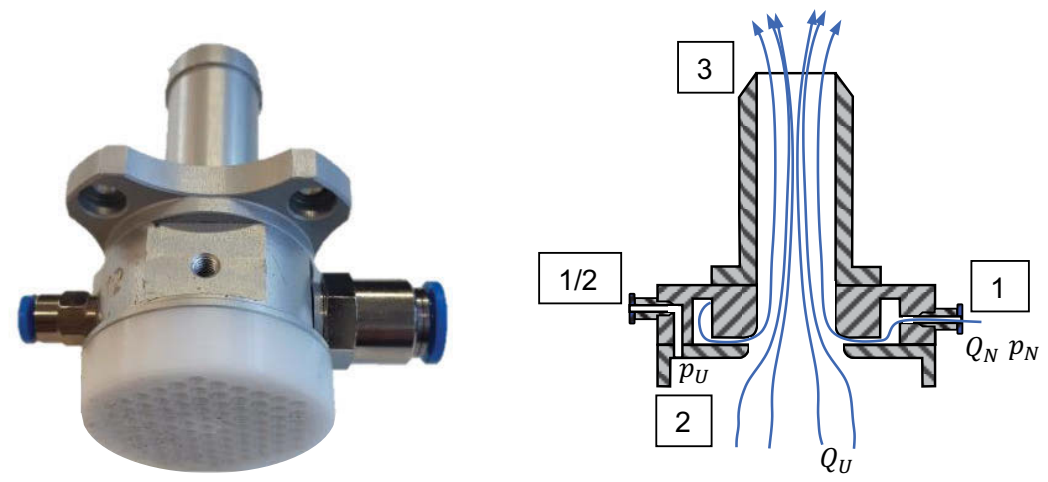

Fig. 2. Left: SCG 1xE100 gripper with suction plate

Right: cross sectional sketch of a coanda-effect based gripper based on [8]

The gripper is activated by supplying air at a positive operating pressure $p_{N}$ to port 1 this high pressure air fills a primary cavity, which it can escape through a thin slit leading to a central secondary cavity. The inwards moving air will tend to adhere to the slightly curved top surface. This primary flow $Q_{N}$ facing upwards will have a suction effect on the air at port 2 creating a negative pressure $p_{U}$ and a secondary airflow $Q_{U}$. During operation an additional suction plate is screwed onto the gripper at this interface. The combined airflow will leave the suction cavity by port 3 . Another connection from the outside directly to the suction cavity is provided by port $1 / 2$, which is usually used for a blow off functionality [5].

Initial tests probing the internal suction pressure at different positions by laterally inserting hypodermic needles into the suction cavity have shown, that even small translational variations of the probing-position can have small (varying by about $0.05 \mathrm{kPa}$ at suction pressures of $p_{U}=0.1 \mathrm{kPa}$ ) but significant effects on the resulting pressure 
readings. These differences are presumably caused by the non-homogenous suction plate geometry and turbulent flow regimes directly behind the it. Therefore, an optimal probing position should be situated as far away from the plate as possible. For transferability of measurements amongst different SCG- 1xE100 grippers the blow-off port 1/2 has been chosen as an ideal probing position.

\section{Procedure}

An adapting gripping system capable of monitoring and reacting to changing disturbance forces may be realized by using the controller architecture shown in Fig. 8.

In such a system a desired contact force $F_{C P \text {,des }}$ can be set and controlled, which will directly implement dynamical reactions to any disturbance forces acting on the handled material (see equation (2)). A PID-controller can be used to dynamically change the operating pressure of a suction gripper depending on an error measurement based on desired and estimated contact forces between the gripper and the handled textiles.

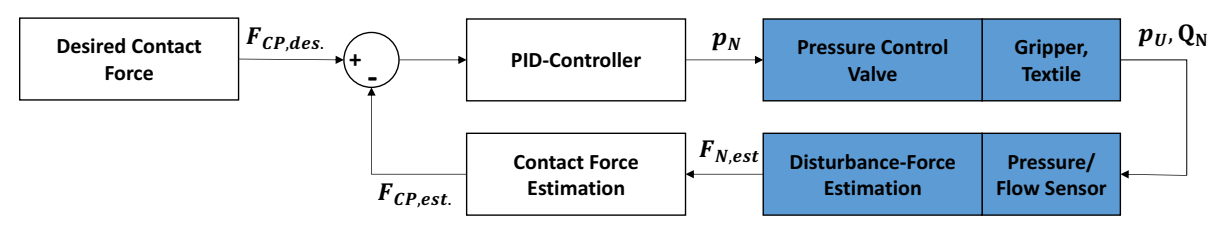

Fig. 3. Proposed control loop for the final gripper system. Parts analyzed in this research are highlighted

This research aims to show how a disturbance force estimation in the shape of a model $F_{e}\left(Q_{N}, p_{U}\right)$ can be obtained by taking the following steps:

1. Recording of example pull-of-experiment data

2. Construction of a mathematical model based on the obtained data

3. Qualitative confirmation of the correlation between measured and estimated disturbance forces by applying the model to further validation tests

\section{Experimental Setup}

In order to characterize the correlation of disturbance forces $F_{N}$ and the suction pressure $p_{U}$ an experiment as shown below is set up.

The experimental setup sketched in Fig. 3 consists of an SCG 1xE100 gripper that can be moved up and down by a distance $s$ with a lead screw driven by an electric stepper motor (not depicted in the figure). The force $F$, at which the gripper is pulled towards the base is measured by a force sensor. The material specimen is clamped between two metal plates with a central rectangular cutout (100x100mm seen in Fig. 3.). The operating pressure $p_{N}$ is supplied by a proportioning valve, while the flow rate $Q_{N}$ is measured by a flow meter and suction pressure $p_{U}$ being monitored by a differential pressure sensor. 


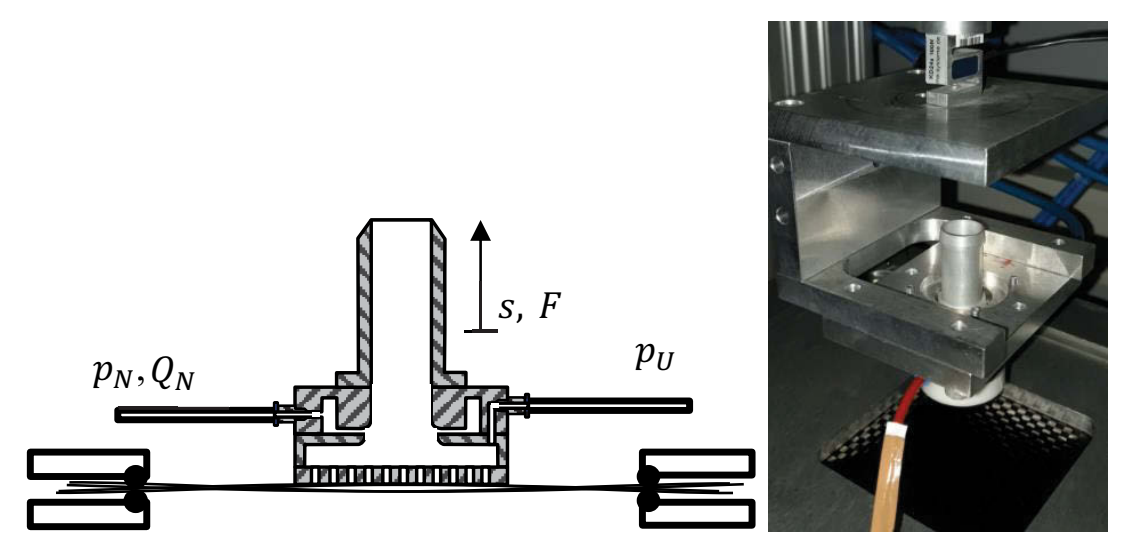

Fig. 4. Sketch and photograph of the used test stand.

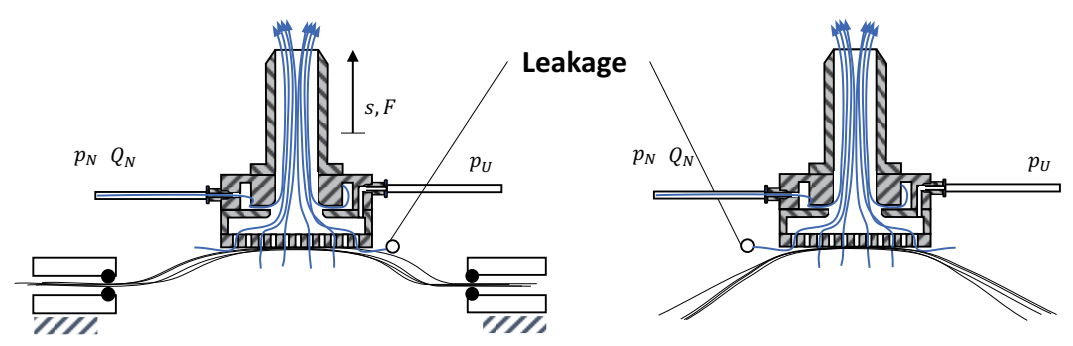

Fig. 5. Leakage occurring after deformation of the gripped fabric

Pull-off tests have been performed by supplying a specific operating pressure $p_{N}$ and moving the gripper upwards by an increasing distance $s$, while recording $Q_{N}, F$ and $p_{U}$. This movement leads to a gradual separation of the material from the suction plate allowing for additional air to bypass the manipulated sheet, avoiding the pressure drop across the textile, leaking into the suction cavity and thereby decreasing the measured value of $p_{U}$. This leakage happens in the test stand as well as when gripping freely hanging sheets as depicted in Fig. 4.

\section{$7 \quad$ Experimental Results}

\subsection{Model Construction}

This section discusses the aforementioned offline calibration for the carbon fiber material shown in Fig. 3.

Exemplary pull-off tests yield the results presented in Fig. 5, with pull-off forces $F$ and differential pressures $p_{U}$ plotted against the traveled distance s (Fig. 5 a). $p_{U}$ and $F$ are plotted against one another from test start up until the maximum force $F_{\max }$ is 
reached (Fig. 5 b). The same plot is repeated on the right for measurements after $F_{\text {max }}$ was reached (Fig. 5 c). The clusters of lines shown in Fig. 5 correlate to supply pressures of $0.5 ; 1 ; 1.5 ; 2 ; 2.5$ and 3 Bar.

a)

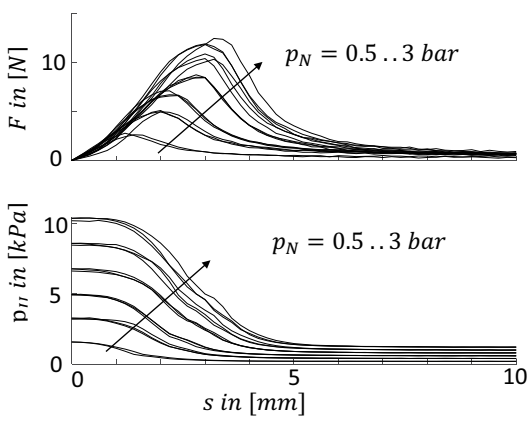

b)

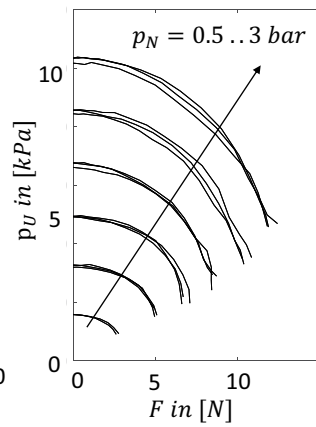

c)

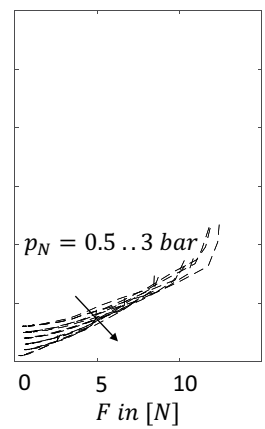

Fig. 6. Measured forces and negative pressures for an exemplary pull-off test

At the beginning of any given pull-off-test the pressure $p_{U}$ stays nearly constant, with no noticeable separation of the gripped material from the suction plate. Recorded forces are here mainly dominated by the material deformation. After $0.5-1.5 \mathrm{~mm}$ of travel slight separation can be observed with a decline in $p_{U}$. The recorded forces keep rising until a maximum force $F_{\max }$ is reached close to an inflection point seen in the $p_{U} \times s$ plot between 1 to $4 \mathrm{~mm}$ of travel. At $\mathrm{s}=\sim 5 \mathrm{~mm}$ full separation can be observed with forces quickly approaching $0 \mathrm{~N}$ and $p_{U}$ settling at a non-zero value generated by the flow resistance of the installed suction plate.

Since the supply pressure at the gripper is set and not directly measured, the operating air flow measurement $Q_{N}$, which behaves almost proportional to $p_{N}$ has proven to be more reliable in dynamic situations. As seen in Fig. 5 the negative pressure increases practically linearly with increasing operating pressure, while decreasing almost quadratically with increasing disturbance forces. Based on this observation a simple polynomial modeling approach $p_{U}\left(F, Q_{N}\right)=p_{00}+p_{10} F+p_{01} Q_{N}+p_{11} F Q_{N}+p_{20} F^{2}$ is chosen. A parameter optimization for the specific results shown in Fig. 5, gives a polynomial numerical model, relating pull off forces and operating pressure to the measured differential pressure (before $F_{\max }$ was reached):

$p_{U, a}\left(F, Q_{N}\right)=-1826 P a-279.8 F \frac{P a}{N}+$
$74.02 Q_{N} \frac{P a}{l m^{-1}}-56.72 F^{2} \frac{P a}{N^{2}}-3.168 F Q_{N} \frac{P a}{N l m^{-1}}$

Here, $Q_{N}$ is given in norm liters per minute.

The model represented by equation (3) reaches an adjusted $\bar{R}^{2}=0.9930$ over 224 sample points collected in 18 individual experiments. 
Analogously, another model can be obtained for the part of the tests after the maximum force has been reached.

$$
\begin{aligned}
& p_{U, b}\left(F, Q_{N}\right)=-301.3 P a-161.5 F \frac{P a}{N}+ \\
& 9.124 Q_{N} \frac{P a}{l \mathrm{~min}^{-1}}+15.64 F^{2} \frac{P a}{N^{2}}-0.8202 F Q_{N} \frac{P a}{N l \mathrm{~min}^{-1}}
\end{aligned}
$$

Using the same approach, similarly close approximations have been found for a variety of different materials. The two models described above can be combined using a linear separator at $p_{U}=\frac{1}{160} \frac{Q_{N}}{\mathrm{lmin}^{-1}} \cdot 4000 \mathrm{~Pa}-600 \mathrm{~Pa}$.

$p_{U}\left(F, Q_{N}\right)=\left\{\begin{array}{l}p_{U, a}\left(F, Q_{N}\right), \text { if } \frac{1}{160} \frac{Q_{N}}{l \mathrm{~min}^{-1}} \cdot 4000 P a-600 P a<p_{U} \\ p_{U, b}\left(F, Q_{N}\right), \text { else }\end{array}\right.$

For any combination of $p_{U}>0$ and $Q_{N}>0$ a corresponding estimated disturbance force $F_{e}$ can be found by calculating the force, which minimizes the absolute difference between the predicted and measured negative pressure:

$F_{e}\left(p_{U}, Q_{N}\right)=\arg \min _{F}\left(\left|p_{U}\left(F, Q_{N}\right)-p_{U}\right|\right)$

\subsection{Model Application}

Manually moving the gripper up and down on the test-stand allows for a comparison between measured forces and estimations made by the previously described models, solely dependent on $p_{U}$ and $Q_{N}$. Results of these simple validation tests at an operating pressure of $p_{N}=2$ bar can be seen in Fig. 6 .

It is clearly visible, that significant disturbance forces and in turn separations are directly seen by the estimation. While both shown curves overlap for most parts of the diagram slight deviations can be seen, which may be explained by non-elastic behavior of the tested fabric.

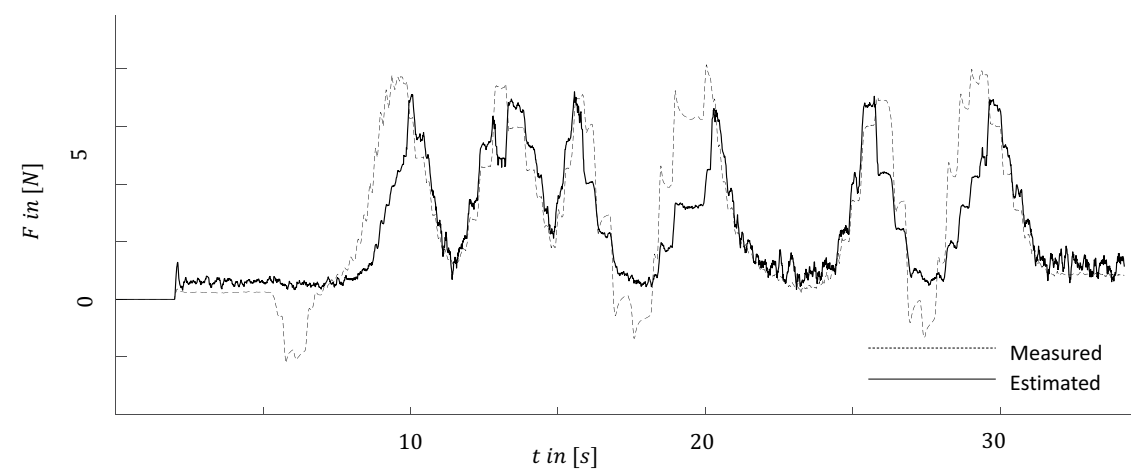

Fig. 7. Model evaluation by manual movement of the gripper 
In another test manually pulling the fabric down similar observations were made as shown in Fig. 7.

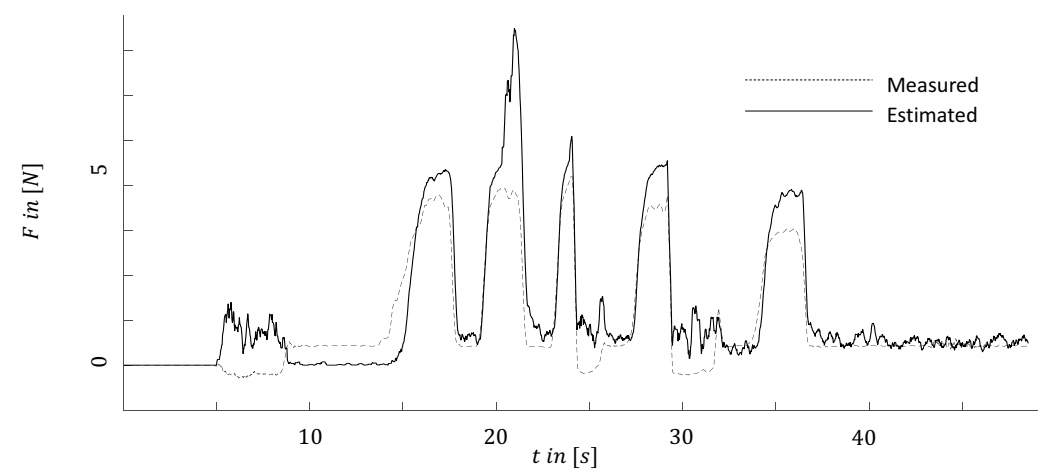

Fig. 8. Model evaluation by manual movement of the fabric

Measured and estimated forces closely match one another while forces are applied evenly around the gripper. Under this load case the model estimation shows an average error of about $1 \mathrm{~N}$.

With punctual loads (as seen at $t=20 \mathrm{~s}$ in Fig. 7) forces will not be estimated as precisely. Here, the estimated force is off by almost $100 \%$. Nevertheless, it can be concluded, that the estimation model qualitatively shows good correlation with the measured data.

\section{Conclusion and Outlook}

This work has introduced a differential pressure-based estimation method for disturbance forces acting on fabric being manipulated by a low-pressure suction gripper. After obtaining experimental data, polynomial models can be formulated which serve as a look-up point for the desired force estimation. It has been shown, that such estimation model can be used to deduce qualitative information about forces acting on the gripped material. While these estimates do not show perfect resemblance of the forces, especially for different load cases, the two show a strong correlation.

In future research these results will be generalized to allow for model estimation based on material properties and gripper characteristics. Which will then be used to define a control structure capable of adjusting the systems. As shown by the works of Förster such a control structure can furthermore be used for robust separation of singular sheets of air-permeable material from a stack magazine [3], paving the way for highly automated production lines in both classical and technical textile industries. 


\section{Acknowledgement}

This research was financially supported by the German Research Foundation (DFG) (funding no. 397485737)

\section{References}

1. Herrmann AS, Pabsch A., Kleinberg M (2000) Kostengünstige Faserverbundstrukturen: eine Frage neuer Produktionsansätze. https://www.dlr.de/fa/Portaldata/17/Resources/dokumente/publikationen/2000/03_herrmann.pdf. Accessed 30 Oct 2019

2. Fleischer J, Ochs A, Koch S-F (2012) Ultrasonic-assisted adhesive handling of limp and air-permeable textile semi-finished products in composites manufacturing. 4th CIRP Conference on Assembly Technologies and Systems: 7-10

3. Förster F (2017) Geregeltes Handhabungssystem zum zuverlässigen und energieeffizienten Handling textiler Kohlenstofffaserzuschnitte. 0724-4967 197

4. Straßer G (2012) Greiftechnologie für die automatisierte Handhabung von technischen Textilien in der Faserverbundfertigung. Zugl.: München, Techn. Univ., Diss., 2011. Forschungsberichte IWB, vol 256. Utz, München

5. J. Schmalz GmbH Operating Instructions Schmalz Composite Gripper. https://pimmedia.schmalz.com/Dokumente/Bedienungsanleitung/10/1001/100130/10013000170/BAL_10.01.30.00170_de-DE_01.pdf. Accessed 04 Oct 2019

6. Claudia Anna Ehinger (2012) Automatisierte Montage von Faserverbund-Vorformlingen. Dissertation, Technische Universität München

7. Brink M, Ohlendorf J-H, Thoben K-D (2018) Development of a Handling System with integrated Sensors for Textile Preforms using Additive Manufacturing. Procedia Manufacturing 24: 114-119. doi: 10.1016/j.promfg.2018.06.016

8. Fleischer J, Förster F, Crispieri NV (2014) Intelligent gripper technology for the handling of carbon fiber material. Production Engineering 8(6): 691-700. doi: 10.1007/s11740014-0549-8

9. Dirk Jodin (1991) Untersuchungen zur Handhabung von biegeweichen Flächenzuschnitten aus Leder mit pneumatischen Greifern. Dissertation, Universität Dortmung

10. Fleischer J, Förster F, Gebhardt J (2016) Sustainable Manufacturing Through Energy Efficient Handling Processes. Procedia CIRP 40: 574-579. doi:

10.1016/j.procir.2016.01.136

11. Fleischer J, Ochs A, Förster F (2013) Gripping Technology for Carbon Fibre Material. CIRP International Conference on Competitive Manufacturing, Band: Green Manufacturing for a Blue Planet: $65-71$

12. Götz R (1991) Strukturierte Planung flexibel automatisierter Montagesysteme für flächige Bauteile. iwb Forschungsberichte, Berichte aus dem Institut für Werkzeugmaschinen und Betriebswissenschaften der Technischen Universität München, vol 39. Springer Berlin Heidelberg, Berlin, Heidelberg, s.l. 
Open Access This chapter is licensed under the terms of the Creative Commons Attribution 4.0 International License (http://creativecommons.org/licenses/by/4.0/), which permits use, sharing, adaptation, distribution and reproduction in any medium or format, as long as you give appropriate credit to the original author(s) and the source, provide a link to the Creative Commons license and indicate if changes were made.

The images or other third party material in this chapter are included in the chapter's Creative Commons license, unless indicated otherwise in a credit line to the material. If material is not included in the chapter's Creative Commons license and your intended use is not permitted by statutory regulation or exceeds the permitted use, you will need to obtain permission directly from the copyright holder. 